\title{
Nanotechnology in Diabetes Management
}

\author{
Lagopati $\mathrm{N}^{1,2 *}$ and Pavlatou $\mathbf{E A}^{1}$ \\ ${ }^{1}$ Laboratory of General Chemistry, School of Chemical Engineering, Greece \\ ${ }^{2}$ Laboratory of Histology-Embryology, Molecular Carcinogenesis Group, Faculty of Medicine, Greece.
}

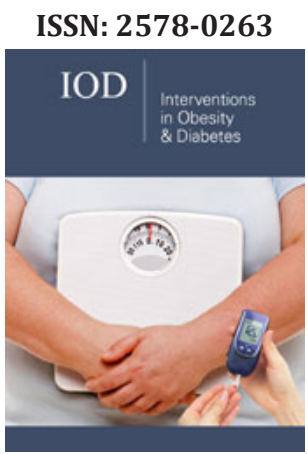

*Corresponding author: Nefeli Lagopati, Laboratory of General Chemistry, School of Chemical Engineering, National Technical University of Athens, Zografou Campus, Athens, Greece

\section{Submission: 侮April 14, 2020}

Published: 僵March 16, 2021

Volume 5 - Issue 1

How to cite this article: Lagopati $\mathrm{N}$, Pavlatou EA. Nanotechnology in Diabetes Management. Interventions Obes Diabetes 5(1). IOD. 000603. 2021.

DOI: $10.31031 /$ IOD.2021.05.000603

Copyright@ Lagopati N. This article is distributed under the terms of the Creative Commons Attribution 4.0 International License, which permits unrestricted use and redistribution provided that the original author and source are credited.

\begin{abstract}
Nanotechnology is an interdisciplinary scientific field with a great number of applications, which are developed in order to improve the quality of life. Nanomedicine is a specialized branch of medicine that applies the fundamentals of nanotechnology to the prevention, diagnosis and treatment of various diseases, such as cancer, cardiovascular diseases and diabetes. Diabetes mellitus is considered to be among the major afflictions of modern western society. The common approach of this condition is a prescribed insulin replacement therapy, including injections of long-acting insulin at mealtimes. Regarding the everyday routine, insulin injections and glucose tests can be painful and time consuming for diabetic patients. Many efforts are given to overcome the drawbacks of injection therapy, but there is the need for new safe and cost-effective technologies for diagnosis and treatment. Nanotechnology has obtained increasing importance in the research of diabetes. Nanotechnology-based tests can provide more accurate information for the diagnosis of diabetes mellitus. Several therapeutic methods have been proposed for non-invasive monitoring of blood glucose, based on nanotechnology. Some representative achievements include the molecular diagnosis of diabetes, the oral delivery of insulin with the use of nanospheres as biodegradable polymeric carriers, the development of artificial beta cells and artificial pancreas. The aim of this review is to provide insights into the role of nanotechnology in diabetes diagnosis and treatment, shedding light on the potential of nanotechnology in this field and discussing the future prospects.
\end{abstract}

Keywords: Nanomedicine; Nanotechnology; Diabetes mellitus; Nanomaterials; Diagnosis; Treatment

\section{Introduction}

Nanotechnology is a scientific and technological combination, integrating various fields, such as physics [1], chemistry [2], biotechnology and engineering [3]. It is considered as the manipulation of matter with at least one dimension sized from 1 to 100 nanometers [4]. The interesting potential of nanotechnology, due to the special properties of nanomaterials, leads to a great number of applications, which are developed in order to improve the quality of life [5]. Nanomedicine is a specialized branch of medicine that applies the fundamentals of nanotechnology to the prevention and/or the treatment of various diseases [6]. Thus, nanomedicine involves the utilization of nanostructured materials for diagnosis, delivery, detection or actuation purposes in a living organism [7]. There are numerous companies specializing in the fabrication of new forms of nanosized matter, with anticipated applications that include medical therapeutics and diagnostics, energy production, molecular computing and structural materials $[4,8]$. Nanotechnology can enhance drug delivery to those areas which were unfavorable for macromolecules to approach [9]. Furthermore, it offers new implantable sensing technologies, providing accurate medical information [10]. Cancer and cardiovascular diseases diagnosis and treatment, dental applications and development of bone implants are among the most famous applications of nanomedicine [11-16]. Diabetes is considered to be among the major afflictions of modern western society. Recent studies demonstrated that around approximately 9.3 percent of the global adult population suffered from diabetes in 2019 [17]. According to mathematical models, based on clinical data, by the year 2045, this percentage is expected to rise to almost 11 percent $[17,18]$. Diabetes is typically characterized by increased thirst, excessive weight loss or excessive desire to eat, increased urge for urination and thus resulting in abnormal increase in blood glucose level $[19,20]$. It is classified as Type 1 , Type 2 or gestational diabetes mellitus, depending on the reason for high blood sugar [19-21]. In type 1-diabetes, the body cannot produce insulin due to loss of $\beta$-cells, as a result of T-cell mediated autoimmune attack [22]. The common approach of this condition is a prescribed insulin replacement therapy, including injections of long-acting insulin at mealtimes [23]. An insulin-resistance combined with insulin deficiency 
is found in patients, suffering from type 2-diabetes [24]. Exercise and regulation of the meals is suggested for the initial treatment of type of diabetes [25]. Diabetes can lead to serious long-term health complications, such as cardiovascular disease, chronic kidney disease, stroke, foot ulcers, damage to the nerves, damage to the eyes and cognitive impairment and is among the top ten leading causes of death worldwide [26,27].

Regarding the everyday routine, insulin injections and glucose tests can be painful and time consuming for diabetic patients [28]. Many efforts are given to overcome the drawbacks of injection therapy [29]. Several technologies have been developed, such as continuous glucose monitors and insulin pumps to improve patient compliance [30]. But there is the need for new safe and costeffective technologies for diagnosis and treatment, since there is still risk of patient's infection and scarring due to implanted sensors and cannulas [31]. All the widely used devices must be frequently replaced and maintained with a high cost for the patients and the health systems worldwide [32]. Nanotechnology has obtained increasing importance in the research of diabetes [33]. It can provide more accurate information for the diagnosis of diabetes mellitus [34]. Furthermore, several therapeutic methods have been proposed for non-invasive monitoring of blood glucose, based on nanotechnology [35]. Some representative achievements include the molecular diagnosis of diabetes, the oral delivery of insulin with the use of nanospheres as biodegradable polymeric carriers, the development of artificial beta cells and artificial pancreas $[34,36]$. Thus, this review outlines the role of nanotechnology in diabetes diagnosis and treatment, shedding light on the potential of nanotechnology in this field and discussing the future prospects.

\section{Nanotechnology and Diabetes Diagnosis}

Nanotechnology can provide sensing technologies for accurate and medical information, for diagnosis of diabetes [37]. Diabetes blood sugar level tests require autonomous periodical checks by the patients, to avoid the risk of blood glucose decrease to dangerous levels [38]. Sometimes this routine is difficult and painful to be held, particularly for the elderly people and the children [39]. Nanotechnology can offer the opportunity for the development of implantable and wearable sensing technologies, providing continuous and accurate medical information [40]. The most common ways of exploiting nanotechnology in the diagnosis of diabetes is by applying microphysiometer or by using implantable sensor [41]. The microphysiometer is built from multiwalled carbon nanotubes, which are electrically conductive [42]. The concentration of insulin in the chamber is directly related to the current at the electrode and thus, the nanotubes are absolutely functionable at $\mathrm{pH}$ levels which are characteristic of living cells [43]. The conventional detection methods typically measure insulin production at intervals, by collecting and measuring small samples, periodically [44]. The microphysiometer can detect insulin levels continuously and indirectly, by estimating the transfer of electrons which are produced when insulin molecules get oxidize, by the glucose [44]. Fundamentally, when the cells produce more insulin molecules, the current which is generated inside the sensor, increases and vice versa, allowing real time monitoring insulin concentrations in [45].

Nanostructured implantable sensors use polyethylene glycol beads, coated with fluorescent molecules in order to monitor diabetes blood sugar levels [46]. The beads are injected under the skin, staying in the interstitial fluid [47]. If the glucose in the interstitial fluid falls to dangerous levels, glucose displaces the fluorescent molecules and creates a glow, which is seen on a tattoo placed on the arm [48]. This method is considered as very effective. However, sensor microchip is another alternative, which is being developed to continuously monitor crucial body parameters such as pulse, blood glucose and temperature [40]. In these applications, the microchip is implanted under the skin, transmitting a signal that could be monitored continuously [40]. Recently a microchipbased test to distinguish between the two main forms of diabetes mellitus, allowing differential diagnosis has been developed [49]. Actually, this cheap, portable, microchip-based test can diagnose type-1 diabetes. Traditional methods for detecting diabetes are expensive, quite slow and they are available only in well-equipped health-care centers [50]. The proposed test applies fluorescence to detect the antibodies. The glass plates which are formed the base of each microchip are coated with gold nanoparticle-sized, allowing the amplification of the fluorescent signal in order to obtain reliable antibody detection [51]. The gold nanoparticles ensure the creation of nanogaps, supporting the enhanced electric field [52]. This technology is expected to improve patient care, assisting in a better understanding of the disease.

\section{Nanomaterials and Diabetes Treatment}

Various types of nanomaterials are currently studied for insulin delivery in diabetes treatment [53]. Ceramic nanoparticles, liposomes, dendrimers, polymeric biodegradable nanoparticles and polymeric micelles are the most promising among the proposed ones [54], (Figure 1). Depending on the type of administration each and every one of these categories of nanomaterials gathers some advantages [4].

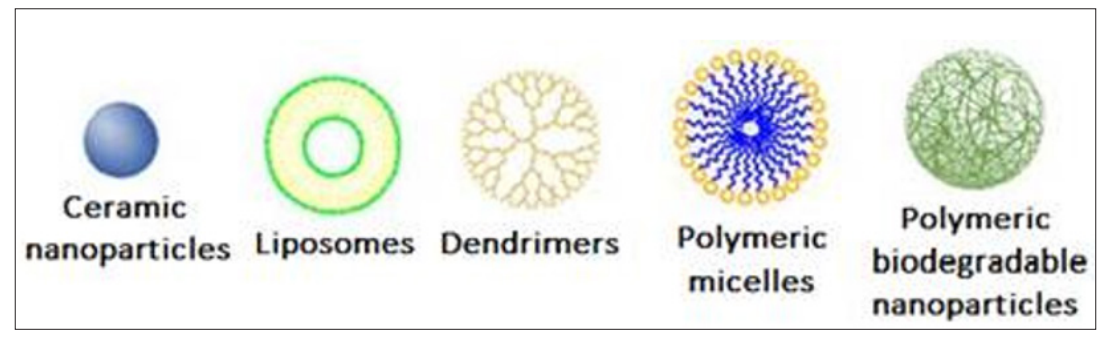

Figure 1: Nanomaterials suitable for diabetes treatment. 


\section{Oral administration}

Oral insulin administration is considered as the most convenient method for diabetes mellitus maintenance [29]. However, the intestinal epithelium is considered as a major barrier to the absorption of hydrophilic drugs, like insulin, as lipid-bilayer cell membranes don't allow the diffusion of these drugs to the bloodstream [55]. Drug delivery systems based on gastric enzymes ensure the transfer and the degradation of the insulin in the stomach [7]. A protective matrix is necessary to embed the active substance, protecting it from the harsh environment inside the stomach [56]. A combination of calcium phosphate-polyethylene glycol-insulin with casein is indicated as an effective choice [57]. Mansoor et al. [58] present polymer-based nanoparticle strategies for insulin delivery, in various forms [58]. Polymeric nanoparticles are considered quite efficient compared to conventional oral and intravenous administration methods which are widely used [59]. In order to form insulin delivery systems, biodegradable, pH-sensitive polymers surrounded by nanoporous membrane are used, allowing controlled release of insulin [58]. In animal studies, the oral delivery of insulin polymeric nanoparticles is achieved through the use of nano-pellets loaded with insulin [58]. $\mathrm{N}$-isopropylacrylamide, polyethylenimine and polymethacrylic acid are some of the polymer-based nanoparticles which are used for oral insulin administration [60]. Also, co-polymers like $\mathrm{N}$, $\mathrm{N}$-dimethylaminoethyl methacrylatem, polyurethanes, polyacrylic acids, polyanhydrides and polyacrylamide are being under investigation in order to be used as insulin carriers [61]. Hydrogels and microspheres can play a double role, acting both as protease inhibitors by protecting the encapsulated insulin from enzymatic degradation within its matrix as well as permeation enhancers by effectively crossing the epithelial layer post oral administration $[62,63]$. Thus, they can effectively carry insulin, providing a promising strategy for oral insulin administration [58].

Additionally, chitosan nanoparticles are proven to enhance the intestinal absorption of insulin to a greater extent than aqueous solutions of chitosan [55]. In particular, insulin loaded chitosan nanoparticles which are coated with mucoadhesive chitosan seem to prolong their residence in the small intestine [64]. These composite nanomaterials can efficiently infiltrate into the mucus layer, mediating transient opening within the tight junctions between epithelial cells, becoming unstable and finally degrading due to their pH sensitivity [65]. Thus, the insulin which is released from the broken-apart nanocomposites can permeate through the paracellular pathway into the bloodstream [66].

\section{Inhalation}

The new nanotechnology-based insulin system is focusing on inhaling the insulin, instead of injecting it, allowing its controlled release into the bloodstream [29]. Compared to the gastrointestinal route, inhaler systems provide the pros of mild environment, including low enzyme concentrations and neutral pH [67]. Various types of inhaler systems can be used to deliver the active products [68]. Dry powder formulations and solutions are among the most common [69]. The encapsulation of insulin within the nanoparticles, allows the inhalation of the dry powder formulation of insulin into the lungs [70]. Insulin degradation is avoided, ensuring the delivery of insulin to the bloodstream. In order to maximize the efficacy, regular lung function tests are required to be applied to the patients, before the treatment, increasing the cost of this approach [29]. Di J et al. [71] proposed a controlled insulin delivery system, based on injectable polymeric nanoparticle-crosslinked network, able to be noninvasively triggered by a Focused Ultrasound System (FUS) [71]. As a matrix material biodegradable poly(lactic-coglycolic acid) (PLGA) was used [71]. They demonstrated that the resulting FUS-activated insulin encapsulated nano-network could regulate blood glucose levels of type 1 diabetic mice in a long-term [71]. For the treatment of type 2-diabetes, chitosan nanoparticles are considered to be suitable for the development of an inhalation delivery system [53]. Since, insulin is a hydrophilic drug, it is difficult to be diffused through intestinal epithelium [72]. Chitosan can enhance the absorption of insulin [73]. Advanced composite nanomaterials, produced by carboxylated chitosan grafted with poly(methylmethacrylate) seem to increase the efficiency of the controlled release of insulin [65].

\section{Nanopump}

The nanopump is a powerful device with many medical applications. It is a tiny volumetric pump with a pair of check valves that is integrated into a Micro-Electromechanical Systems (MEMS) or a Nano Electromechanical Systems (NEMS) chip [74]. From as structural point of view, the chip is a stack of three layers bonded together. The first one is a Silicon-on- Insulator (SOI) layer with micromachined pump-structures, and the two others are Pyrex cover plates. Insulin delivery is the main application of the pump, introduced by Debiotech [75]. The pump can inject insulin to the patient's body in a constant rate, balancing the amount of glucose in the blood. It can also administer small drug doses over a long period of time [76].

\section{Artificial pancreas}

The development of an artificial pancreas system, comprising of a continuous glucose monitor, glucose meter and an insulin infusion pump for the monitor calibration could be the permanent solution for the patients who suffer from diabetes mellitus [77]. The original initial idea was first demonstrated in 1974 [78]. The fundamental of this concept includes a sensor electrode which can repeatedly measure the level of blood glucose, with the data feeding into a tiny computer [44]. This process can trigger an infusion pump, and the appropriate units of insulin can enter the bloodstream from a small reservoir [79]. The utilization of a tiny silicon box, containing pancreatic beta cells obtained from animals is an alternative approach [80]. This application is used to protect transplanted cells from the immune system. It also allows the sufficient diffusion of glucose, insulin and oxygen [81]. It can be implanted under the skin of diabetes patients. This box is encapsulated in a material with a specific nanopore size. These pores allow glucose and insulin to pass through them, while impede the passage of much larger immune 
system molecules [82]. A smart insulin patch is the promising achievement for insulin delivery [83]. This device can release depending on the body's needs and therefore it is called "smart" [84]. It contains a pack of more than 100 microneedles, which are packed with insulin and glucose-sensing enzymes [85]. The current scientific attempt includes the development of a nanorobot with glucose level sensors on the surface and insulin departed in inner chambers. The sensors on the surface can record any increase in blood glucose levels, triggering selective insulin release [86].

\section{Conclusion}

The impact of nanotechnology on medicine is uncontested. In this manuscript the use of nanotechnology in diabetes diagnosis and treatment was discussed. It was demonstrated that it is very promising in detection of insulin and blood glucose but also in insulin efficient administration and delivery. Nanotechnologybased techniques are being helpful in the development of new strategy for the treatment of diabetes, including glucose-responsive insulin therapy. Continuous glucose monitoring devices as well as insulin delivery systems like artificial pancreas will be invaluable for diabetic patients. Nanotechnology promised a total absence of lag time between glucose detection and insulin delivery, avoiding dangerous situations, such as hypoglycemia. The next generation nanocomposites-mediated insulin in parallel with advanced nanodevices are expected to improve everyday life of diabetic patients in the future.

\section{References}

1. Tamirat $Y$ (2017) The role of nanotechnology in semiconductor industry: Review article. Journal of Materials Science and Nanotechnology 5(2): $1-10$.

2. Shinn E, Hübler A, Lyon D, Perdekamp MG, Bezryadin A, et al. (2013) Nuclear energy conversion with stacks of graphene nanocapacitors. Complexity 18(3): 24-27.

3. Nagamune $T$ (2017) Biomolecular engineering for nanobio/ bionanotechnology. Nano Convergence 4(1): 9 .

4. Jeevanandam J, Barhoum A, Chan YS, Dufresne A, Danquah MK (2018) Review on nanoparticles and nanostructured materials: history, sources, toxicity and regulations. Beilstein Journal of Nanotechnology 9: 10501074

5. Ventola CL (2012) The nanomedicine revolution: part 1: Emerging concepts. P \& T: A Peer-Reviewed Journal for Formulary Management 37(9): 512-525.

6. Soares S, Sousa J, Pais A, Vitorino C (2018) Nanomedicine: Principles, properties, and regulatory issues. Frontiers in Chemistry 6: 360

7. Patra JK, Das G, Fraceto LF, Campos E, Rodriguez Torres M, et al. (2018) Nano based drug delivery systems: Recent developments and future prospects. Journal of Nanobiotechnology 16(1): 71.

8. Vasile C (2018) Polymeric nanocomposites and nanocoatings for food packaging: A review. Materials (Basel, Switzerland) 11(10): 1834.

9. Vega Vásquez P, Mosier NS, Irudayaraj J (2020) Nanoscale drug delivery systems: From medicine to agriculture. Frontiers in Bioengineering and Biotechnology 8: 79.

10. Guk K, Han G, Lim J, Jeong K, Kang T, et al. (2019) Evolution of wearable devices with real-time disease monitoring for personalized healthcare. Nanomaterials (Basel, Switzerland) 9(6): 813.
11. Smith WR, Hudson PW, Ponce BA, Rajaram Manoharan SR (2018) Nanotechnology in orthopedics: A clinically oriented review. BMC Musculoskeletal Disorders 19(1): 67.

12. Martín Giménez VM, Kassuha DE, Manucha W (2017) Nanomedicine applied to cardiovascular diseases: Latest developments. Therapeutic Advances in Cardiovascular Disease 11(4): 133-142.

13. Narang RS, Narang JK (2015) Nanomedicines for dental applicationsscope and future perspective. International Journal of Pharmaceutical Investigation 5(3): 121-123.

14. Lagopati N, Tsilibary EP, Falaras P, Papazafiri P, Pavlatou EA, et al. (2014) Effect of nanostructured $\mathrm{TiO}_{2}$ crystal phase on photoinduced apoptosis of breast cancer epithelial cells. International Journal of Nanomedicine 9: 3219-3230.

15. Lagopati N, Kitsiou PV, Kontos AI, Venieratos P, Kotsopoulou E, et al. (2010) Photo-induced treatment of breast epithelial cancer cells using nanostructured titanium dioxide solution. Journal of Photochemistry and Photobiology A: Chemistry 214(2-3): 215-223.

16. Vamvakas I, Lagopati N, Andreou M, Sotiropoulos M, Gatzis A, et al. (2009) Patient specific computer automated dosimetry calculations during therapy with ${ }^{111}$ In octreotide. European Journal of Radiography 1(4): 180-183.

17. Saeedi P, Petersohn I, Salpea P, Malanda B, Karuranga S, et al. (2019) Global and regional diabetes prevalence estimates for 2019 and projections for 2030 and 2045: Results from the International Diabetes Federation Diabetes Atlas, ( ${ }^{\text {th }}$ edn). Diabetes Research and Clinical Practice 157: 107843.

18. Cho NH, Shaw JE, Karuranga S, Huang Y, da Rocha Fernandes JD, et al. (2018) IDF diabetes atlas: Global estimates of diabetes prevalence for 2017 and projections for 2045. Diabetes Research and Clinical Practice 138: $271-281$

19. Ley SH, Hamdy O, Mohan V, Hu FB (2014) Prevention and management of type 2 diabetes: Dietary components and nutritional strategies. Lancet 383(9933): 1999-2007.

20. Atkinson MA, Eisenbarth GS, Michels AW (2014) Type 1 diabetes. Lancet 383(9911): 69-82.

21. Morgana Dirar A, Doupis J (2017) Gestational diabetes from A to Z. World Journal of Diabetes 8(12): 489-511.

22. Burrack AL, Martinov T, Fife BT (2017) T cell-mediated beta cell destruction: Autoimmunity and alloimmunity in the context of type 1 diabetes. Frontiers in Endocrinology 8: 343.

23. Silver B, Ramaiya K, Andrew SB, Fredrick O, Bajaj S, et al. (2018) EADSG guidelines: Insulin therapy in diabetes. Diabetes Therapy 9(2): 449-492.

24. Cantley J, Ashcroft FM (2015) Q\&A: insulin secretion and type 2 diabetes: Why do $\beta$-cells fail? BMC Biology 13: 33.

25. Colberg SR (2017) Key points from the updated guidelines on exercise and diabetes. Frontiers in Endocrinology 8: 33.

26. Nickerson HD, Dutta S (2012) Diabetic complications: Current challenges and opportunities. Journal of Cardiovascular Translational Research 5(4): 375-379.

27. Cade WT (2008) Diabetes-related microvascular and macrovascular diseases in the physical therapy setting. Physical Therapy 88(11): 13221335.

28. Ballout RA, Arabi A (2017) Painful and prolonged muscle cramps following insulin injections in a patient with type 2 diabetes mellitus: Revisiting the 1992 duke case. Frontiers in Endocrinology 8: 243.

29. Shah RB, Patel M, Maahs DM, Shah VN (2016) Insulin delivery methods: Past, present and future. International Journal of Pharmaceutical Investigation 6(1): 1-9. 
30. Gómez AM, Henao Carrillo DC, Muñoz Velandia OM (2017) Devices for continuous monitoring of glucose: Update in technology. Medical Devices (Auckland, N Z) 10: 215-224.

31. VanEpps JS, Younger JG (2016) Implantable device-related infection. Shock 46(6): 597-608.

32. Adu MD, Malabu UH, Malau Aduli A, Malau Aduli BS (2019) Enablers and barriers to effective diabetes self-management: A multi-national investigation. PLOS ONE 14(6): e0217771.

33. DiSanto RM, Subramanian V, Gu Z (2015) Recent advances in nanotechnology for diabetes treatment. Wiley Interdisciplinary Reviews Nanomedicine and Nanobiotechnology 7(4): 548-564.

34. Veiseh O, Tang BC, Whitehead KA, Anderson DG, Langer R (2015) Managing diabetes with nanomedicine: challenges and opportunities. Nature Reviews Drug Discovery 14(1): 45-57.

35. Makaram P, Owens D, Aceros J (2014) Trends in nanomaterial-based noninvasive diabetes sensing technologies. Diagnostics (Basel, Switzerland) $4(2):$ 27-46.

36. Hu S, De Vos P (2019) Polymeric approaches to reduce tissue responses against devices applied for islet-cell encapsulation. Frontiers in Bioengineering and Biotechnology 7: 134.

37. Taguchi M, Ptitsyn A, McLamore ES, Claussen JC (2014) Nanomaterialmediated biosensors for monitoring glucose. Journal of Diabetes Science and Technology 8(2): 403-411.

38. Duggan EW, Carlson K, Umpierrez GE (2017) Perioperative hyperglycemia management: An update. Anesthesiology 126(3): 547-560.

39. Bahendeka S, Kaushik R, Swai AB, Otieno F, Bajaj S, et al. (2019) EADSG guidelines: Insulin storage and optimisation of injection technique in diabetes management. Diabetes Therapy: Research, Treatment and Education of Diabetes and Related Disorders 10(2): 341-366.

40. Dias D, Cunha PSJ (2018) Wearable health devices-vital sign monitoring, systems and technologies. Sensors (Basel, Switzerland) 18(8): 2414.

41. Rauf S, Hayat Nawaz MA, Badea M, Marty JL, Hayat A (2016) Nanoengineered biomimetic optical sensors for glucose monitoring in diabetes. Sensors (Basel, Switzerland) 16(11): 1931.

42. Ramachandran K, Raj Kumar T, Babu KJ, Gnana Kumar G (2016) Ni-Co bimetal nanowires filled multiwalled carbon nanotubes for the highly sensitive and selective non-enzymatic glucose sensor applications. Scientific Reports 6: 36583.

43. Villena Gonzales W, Mobashsher AT, Abbosh A (2019) The progress of glucose monitoring-A review of invasive to minimally and non-invasive techniques, devices and sensors. Sensors (Basel, Switzerland) 19(4): 800.

44. Bruen D, Delaney C, Florea L, Diamond D (2017) Glucose sensing for diabetes monitoring: Recent developments. Sensors (Basel, Switzerland) 17(8): 1866.

45. Röder PV, Wu B, Liu Y, Han W (2016) Pancreatic regulation of glucose homeostasis. Experimental \& Molecular Medicine 48(3): e219.

46. Chen C, Zhao XL, Li ZH, Zhu ZG, Qian SH, et al. (2017) Current and emerging technology for continuous glucose monitoring. Sensors (Basel, Switzerland) 17(1): 182 .

47. Samant PP, Prausnitz MR (2018) Mechanisms of sampling interstitial fluid from skin using a microneedle patch. Proceedings of the National Academy of Sciences of the United States of America 115(18): 45834588 .

48. Srivastava R, Jayant RD, Chaudhary A, McShane MJ (2011) "Smart tattoo" glucose biosensors and effect of coencapsulated anti-inflammatory agents. Journal of Diabetes Science and Technology 5(1): 76-85.

49. Mannino GC, Andreozzi F, Sesti G (2019) Pharmacogenetics of type 2 diabetes mellitus, the route toward tailored medicine. Diabetes/ Metabolism Research and Reviews 35(3): e3109.
50. Kahanovitz L, Sluss PM, Russell SJ (2017) Type 1 diabetes-A clinical perspective. Point of Care 16(1): 37-40.

51. Chinen AB, Guan CM, Ferrer JR, Barnaby SN, Merkel TJ, et al. (2015) Nanoparticle probes for the detection of cancer biomarkers, cells, and tissues by fluorescence. Chemical Reviews 115(19): 10530-10574.

52. Kohout C, Santi C, Polito L (2018) Anisotropic gold nanoparticles in biomedical applications. International Journal of Molecular Sciences 19(11): 3385.

53. Souto EB, Souto SB, Campos JR, Severino P, Pashirova TN, et al. (2019) Nanoparticle delivery systems in the treatment of diabetes complications. Molecules (Basel, Switzerland) 24(23): 4209.

54. Singh AP, Biswas A, Shukla A, Maiti P (2019) Targeted therapy in chronic diseases using nanomaterial-based drug delivery vehicles. Signal Transduction and Targeted Therapy 4: 33.

55. Yang NJ, Hinner MJ (2015) Getting across the cell membrane: An overview for small molecules, peptides, and proteins. Methods in Molecular Biology (Clifton, N J) 1266: 29-53.

56. Date AA, Hanes J, Ensign LM (2016) Nanoparticles for oral delivery: Design, evaluation and state-of-the-art. Journal of Controlled Release 240: 504-526.

57. Trofimov AD, Ivanova AA, Zyuzin MV, Timin AS (2018) Porous inorganic carriers based on silica, calcium carbonate and calcium phosphate for controlled/modulated drug delivery: Fresh outlook and future perspectives. Pharmaceutics 10(4): 167.

58. Mansoor S, Kondiah P, Choonara YE, Pillay V (2019) Polymer-based nanoparticle strategies for insulin delivery. Polymers 11(9): 1380.

59. Rizvi S, Saleh AM (2018) Applications of nanoparticle systems in drug delivery technology. Saudi Pharmaceutical Journal 26(1): 64-70.

60. Li J, Cai C, Li J, Li J, Li J, et al. (2018) Chitosan-based nanomaterials for drug delivery. Molecules (Basel, Switzerland) 23(10): 2661.

61.Wu JZ, Yang Y, Li S, Shi A, Song B, et al. (2019) Glucose-sensitive nanoparticles based on poly(3-acrylamidophenylboronic acid-block$\mathrm{N}$-vinylcaprolactam) for insulin delivery. International Journal of Nanomedicine 14: 8059-8072.

62. Wagner AM, Gran MP, Peppas NA (2018) Designing the new generation of intelligent biocompatible carriers for protein and peptide delivery. Acta Pharmaceutica Sinica B 8(2): 147-164.

63. Yu J, Zhang Y, Bomba H, Gu Z (2016) Stimuli-responsive delivery of therapeutics for diabetes treatment. Bioengineering \& Translational Medicine 1(3): 323-337.

64. Marques C, Som C, Schmutz M, Borges O, Borchard G (2020) How the lack of chitosan characterization precludes implementation of the safeby-design concept. Frontiers in Bioengineering and Biotechnology 8: 165.

65. Sharma G, Sharma AR, Nam JS, Doss GP, Lee SS, et al. (2015) Nanoparticle based insulin delivery system: the next generation efficient therapy for type 1 diabetes. Journal of Nanobiotechnology 13: 74.

66. Homayun B, Lin X, Choi HJ (2019) Challenges and recent progress in oral drug delivery systems for biopharmaceuticals. Pharmaceutics 11(3): 129.

67. Bajracharya R, Song JG, Back SY, Han HK (2019) Recent advancements in non-invasive formulations for protein drug delivery. Computational and Structural Biotechnology Journal 17: 1290-1308.

68. Ibrahim M, Verma R, Garcia Contreras L (2015) Inhalation drug delivery devices: technology update. Medical Devices (Auckland, N Z) 8: 131-139.

69.Xia Y, Su Y, Wang Q Yang C, Tang B, et al. (2019) Preparation, characterization, and pharmacodynamics of insulin-loaded fumaryl diketopiperazine microparticle dry powder inhalation. Drug Delivery 26(1): $650-660$. 
70. El Sherbiny IM, El Baz NM, Yacoub MH (2015) Inhaled nano-and microparticles for drug delivery. Global Cardiology Science \& Practice 2015: 2.

71. Di J, Price J, Gu X, Jiang X, Jing Y, et al. (2014) Ultrasound-triggered regulation of blood glucose levels using injectable nano-network. Advanced Healthcare Materials 3(6): 811-816.

72. Cao SJ, Xu S, Wang HM, Ling Y, Dong J, et al. (2019) Nanoparticles: Oral delivery for protein and peptide drugs. AAPS PharmSciTech 20(5): 190.

73. Jaafar MHM, Hamid KA (2019) Chitosan-coated alginate nanoparticles enhanced absorption profile of insulin via oral administration. Current Drug Delivery 16(7): 672-686.

74. Barkam S, Saraf S, Seal S (2013) Fabricated micro-nano devices for in vivo and in vitro biomedical applications. Wiley Interdisciplinary Reviews-Nanomedicine and Nanobiotechnology 5(6): 544-568.

75. McAdams BH, Rizvi AA (2016) An overview of insulin pumps and glucose sensors for the generalist. Journal of Clinical Medicine 5(1): 5.

76. Shirin A, Della Rossa F, Klickstein I, Russell J, Sorrentino F (2019) Optimal regulation of blood glucose level in type I diabetes using insulin and glucagon. Plos One 14(3): e0213665.

77. Allen N, Gupta A (2019) Current diabetes technology: Striving for the artificial pancreas. Diagnostics (Basel, Switzerland) 9(1): 31.

78. Cobelli C, Renard E, Kovatchev B (2011) Artificial pancreas: past, present, future. Diabetes 60(11): 2672-2682.
79. Gradel A, Porsgaard T, Lykkesfeldt J, Seested T, Gram Nielsen S, et al. (2018) Factors affecting the absorption of subcutaneously administered insulin: Effect on variability. Journal of Diabetes Research 2018: 1205121.

80. Cito M, Pellegrini S, Piemonti L, Sordi V (2018) The potential and challenges of alternative sources of $\beta$ cells for the cure of type 1 diabetes. Endocrine Connections 7(3): R114-R125.

81. Sneddon JB, Tang Q, Stock P, Bluestone JA, Roy S, et al. (2018) Stem cell therapies for treating diabetes: Progress and remaining challenges. Cell Stem Cell 22(6): 810-823.

82. Barkai U, Rotem A, De Vos P (2016) Survival of encapsulated islets: More than a membrane story. World Journal of Transplantation 6(1): 69-90.

83. Veiseh O, Langer R (2015) Diabetes: A smart insulin patch. Nature 524(7563): 39-40.

84. Liu D, Yang F, Xiong F, Gu N (2016) The smart drug delivery system and its clinical potential. Theranostics 6(9): 1306-1323.

85. Yu J, Zhang Y, Ye Y, DiSanto R, Sun W, et al. (2015) Microneedle-array patches loaded with hypoxia-sensitive vesicles provide fast glucoseresponsive insulin delivery. Proceedings of the National Academy of Sciences of the United States of America 112(27): 8260-8265.

86. Malhotra P, Shahdadpuri N (2019) Nano robots for continuous blood glucose diagnosis. International Journal of Trend in Scientific Research and Development 3(6): 1023-1028. 